\title{
Analysis of displayable depth range for retinal projection type super multi-view 3D head-mounted display using the time division projection optical system
}

Hiroaki Maeda, Junya Kohno, Kayo Yoshimoto, Hideya Takahashi

\begin{tabular}{|c|l|}
\hline Citation & Proceedings Volume 10942, Advances in Display Technologies IX; 109420X \\
\hline Date & 2019-03-01 \\
\hline Event & SPIE OPTO, 2019, San Francisco, California, United States \\
\hline Type & Conference Paper \\
\hline Textversion & Author \\
\hline Rights & $\begin{array}{l}\text { C } 2019 \text { Society of Photo-Optical Instrumentation Engineers (SPIE). One print or } \\
\text { electronic copy may be made for personal use only. Systematic reproduction and } \\
\text { distribution, duplication of any material in this publication for a fee or for } \\
\text { commercial purposes, and modification of the contents of the publication are } \\
\text { prohibited. } \\
\text { The following paper is postprint of the publication. After it is published, it will be } \\
\text { found at https://doi.org/10.1117/12.2506983. }\end{array}$ \\
\hline DOI & \begin{tabular}{l}
$10.1117 / 12.2506983$ \\
\hline
\end{tabular}
\end{tabular}

Self-Archiving by Author(s) Placed on: Osaka City University

Hiroaki Maeda, Junya Kohno, Kayo Yoshimoto, and Hideya Takahashi "Analysis of displayable depth range for retinal projection type super multi-view 3D head-mounted display using the time division projection optical system", Proc. SPIE 10942, Advances in Display Technologies IX, 109420X (1 March 2019); https://doi.org/10.1117/12.2506983 


\title{
Analysis of displayable depth range for retinal projection type super multi-view 3D head-mounted display using the time division projection optical system
}

\author{
Hiroaki Maeda ${ }^{a}$, Junya Kohno ${ }^{a}$, Kayo Yoshimoto ${ }^{a}$, and Hideya Takahashi ${ }^{* a}$ \\ aDept. of Electrical and Information Engineering, Graduate School of Engineering, Osaka City Univ., \\ 3-3-138 Sugimoto, Sumiyoshi-ku, Osaka 558-8585, Japan;
}

\begin{abstract}
We have previously proposed the retinal projection type super multi-view head-mounted display (HMD) using the time division projection optical system. By using the super multi-view HMD, it is possible to provide the viewer with the 3D image that induces the accommodation of a human eye naturally. In order to realize the super multi-view method, it is necessary to simultaneously project multiple parallax images on the retina. However, when the parallax between each parallax image is large and the corresponding pixels of each parallax image on the retina do not overlap each other, the retinal image is observed as multiple projection images, therefore the accommodation cannot be induced. In this paper, to estimate the displayable range of 3D image of the proposed super multi-view HMD, we revealed the relationship between the number of parallax images and the displayable range of 3D image by using Gullstrand's schematic eye model and the analysis of the light intensity distributions of retinal images. In order to verify the displayable range of 3D image, we constructed the prototype HMD which projected five parallax images on the retina. We confirmed that the displayable range of 3D image of the prototype HMD is from $900 \mathrm{~mm}$ to $2000 \mathrm{~mm}$ in front of human eye. By clarifying the relationship between the number of parallax images and the displayable range of 3D image, it is possible to freely design the display range of 3D image of the retinal projection type super multi-view HMD.
\end{abstract}

Keywords: head-mounted display, retinal projection display, super multi-view, 3D display

\section{INTRODUCTION}

Mixed Reality technology ${ }^{1}$ has recently been proposed as an approach for practical use of Virtual Reality technology ${ }^{2}$. See-through head-mounted displays (HMD) provide an effective capability for Mixed Reality. By using a see-through HMD, a viewer can see both real world and virtual world at the same time. Some conventional see-through HMDs have been developed. They can display two-dimensional (2D) virtual information. However, when 2D virtual information is displayed by a HMD, a viewer is hard to understand that it is related with a real-world object. What a viewer sees needs to be augmented by three-dimensional (3D) virtual information image in accordance with the real object. In order to overcome this problem, we propose a retinal projection type super multi-view $\mathrm{HMD}^{3-7}$. The smooth motion parallax provided by the super multi-view technique ${ }^{8}$ enables a precise superposition of virtual 3D images on real objects. Moreover, if a viewer focuses his or her eyes on the displayed 3D image, the stimulus for the accommodation of the human eye is produced naturally by the super multi-view technique. Therefore, although proposed HMD is a monocular HMD, it provides viewer with natural 3D images. In addition, proposed retinal projection type super multi-view HMD use the principle of the Maxwellian view. In the Maxwellian view, parallel rays are converged at the center of the pupil, and projected onto the retina directly. Thus, proposed HMD can provide an extreme long focal depth image, and a provided 3D image by proposed HMD is clear and high contrast. However, super multi-view HMD has a problem. The problem is the restricted displayable depth range of 3D images. When the amount of positional gap between corresponding pixels of each parallax image on the retina is too large, a viewer cannot recognize the superimposed parallax image instead of the blurred image. Thus, a viewer cannot see the 3D image since stimulus of accommodation of the human eye isn't induced. In order to estimate the displayable depth range of the 3D image by the super multi-view HMD, we revealed the relationship between the number of parallax images and the displayable depth range of 3D image by using Gullstrand's schematic eye model and the analysis of the light intensity distributions of retinal images. This paper describes the relationship between

*hideya@elec.eng.osaka-cu.ac.jp; 
the number of parallax images and the displayable depth range of 3D image. In Section 2, the principle of retinal projection type super multi-view HMD is described. In Section 3, we describe the relationship between the number of parallax images and the displayable range of 3D image. In Section 4, to verify the effectiveness of the proposed analysis method, we describe experimental result. Finally, we give some conclusions in Section 5.

\section{RETINAL PROJECTION TYPE SUPER MULTI-VIEW HMD}

We have previously proposed the retinal projection type super multi-view 3D head-mounted display using the time division projection optical system. In this paper, since we will clarify the relationship between the number of parallax images and the displayable range of 3D image by the super multi-view HMD, the previously proposed see-through retinal projection type 3D HMD is described here again.

\subsection{Principle of the Maxwellian View}

Figure 1 shows the principle of the Maxwellian view ${ }^{9}$. The transparent LCD is irradiated with parallel rays. The LCD is located on the front focus plane of the lens, and the pupil of an eye is located on the back focus plane of the lens. In this case, an extremely long focal depth image can be seen without causing ocular accommodation.

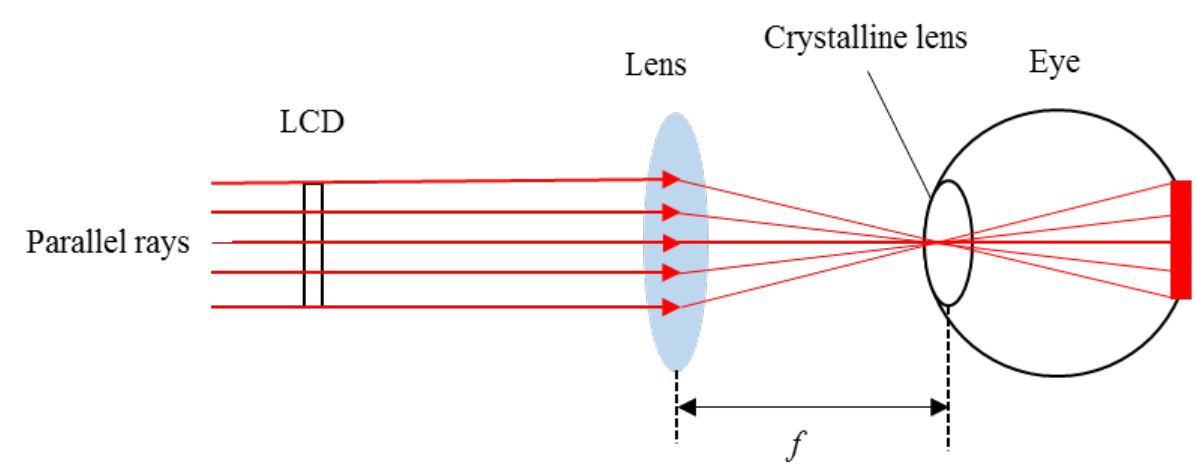

Figure 1. Principle of the Maxwellian view.

\subsection{Super-multi-view}

In the real world, usually illumination light is diffusely reflected at an object's surface and reflected light causes stimulus of accommodation of the eye, as shown in Figure 2(a). In the ray reconstruction method, several pixels that have been angularly multiplexed can be utilized to give the impression that rays are emanating from a converging point, as shown in Figure 2(b). However, multiple rays of the light diffusing at the object's surface are sampled discretely. If the sampling interval of parallax rays is narrower than the pupil's diameter, two or more rays always pass through the pupil of each eye. This condition is called 'super multi-view'. In the super multi-view condition, reconstructed 3D images have very smooth motion parallax, and if a viewer focuses his or her eyes on the reconstructed object's surface, the stimulus for the accommodation of the human eye is reproduced naturally. Therefore, the retinal projection HMD provides viewers with natural 3D virtual images so long as the super multi-view condition is satisfied. In Figure 2(b), each pixel which reconstructs a ray is a corresponding point of each parallax image on the display panel. It is possible to freely set the depth position of the virtual 3D point (image) by changing the parallax of the parallax images as shown in Figures 2(b) and 2(c). 


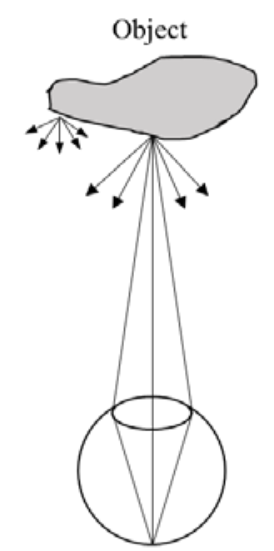

Eye

(a)

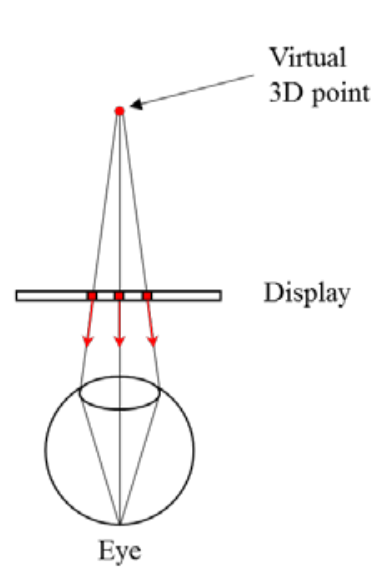

(b)

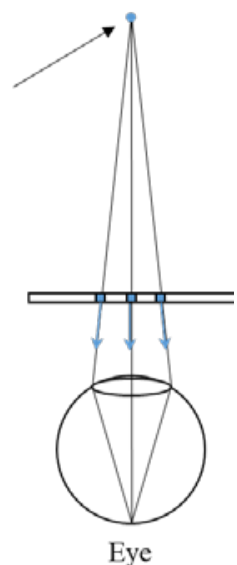

(c)

Figure 2. Schematic diagram of the stimulus of accommodation: (a) real world, (b) and (c) super multi-view.

\subsection{Principle of super multi-view HMD}

Super multi-view HMD uses Holographic optical element (HOE) which forms some convergence point and the principle of the Maxwellian view. As shown in Figure 3, the super multi-view condition is realized by converging multiple parallax images to different positions on the pupil. For the sake of simplicity, here we limit the number of parallax images to three. The parallax image \#i converges on the convergence point $C_{i} . P_{i}$ and $Q_{i}$ denote pixels of the parallax image \#i which correspond to virtual 3D points P and Q, respectively. As shown in Figure 4(a), when the viewer's eye accommodates to the spatial position $P$, the projected images of the pixels $P_{1}, P_{2}$ and $P_{3}$ are focused to the same position on the retina. The viewer feels this image as the $3 \mathrm{D}$ point at the distance $\mathrm{L}_{\mathrm{p}}$. On the other hand, the projected images of the pixels $\mathrm{Q}_{1}, \mathrm{Q}_{2}$ and $\mathrm{Q}_{3}$ are focused to different positions on the retina. The viewer feels this image as the blurred point at the distance $\mathrm{L}_{\mathrm{Q}}$. As shown in Figure 4(b), when the viewer's eye accommodates to the spatial position Q, the projected images of the pixels $\mathrm{Q}_{1}, \mathrm{Q}_{2}$ and $\mathrm{Q}_{3}$ are focused to the same position on the retina and the pixels $\mathrm{P}_{1}, \mathrm{P}_{2}$ and $\mathrm{P}_{3}$ are focused to different positions on the retina. The viewer feels that the image of $\mathrm{Q}$ is in focus and the image of $\mathrm{P}$ is out of focus. Therefore, the super multiview HMD can display the virtual image at the distance within the ability for focusing on the eye.

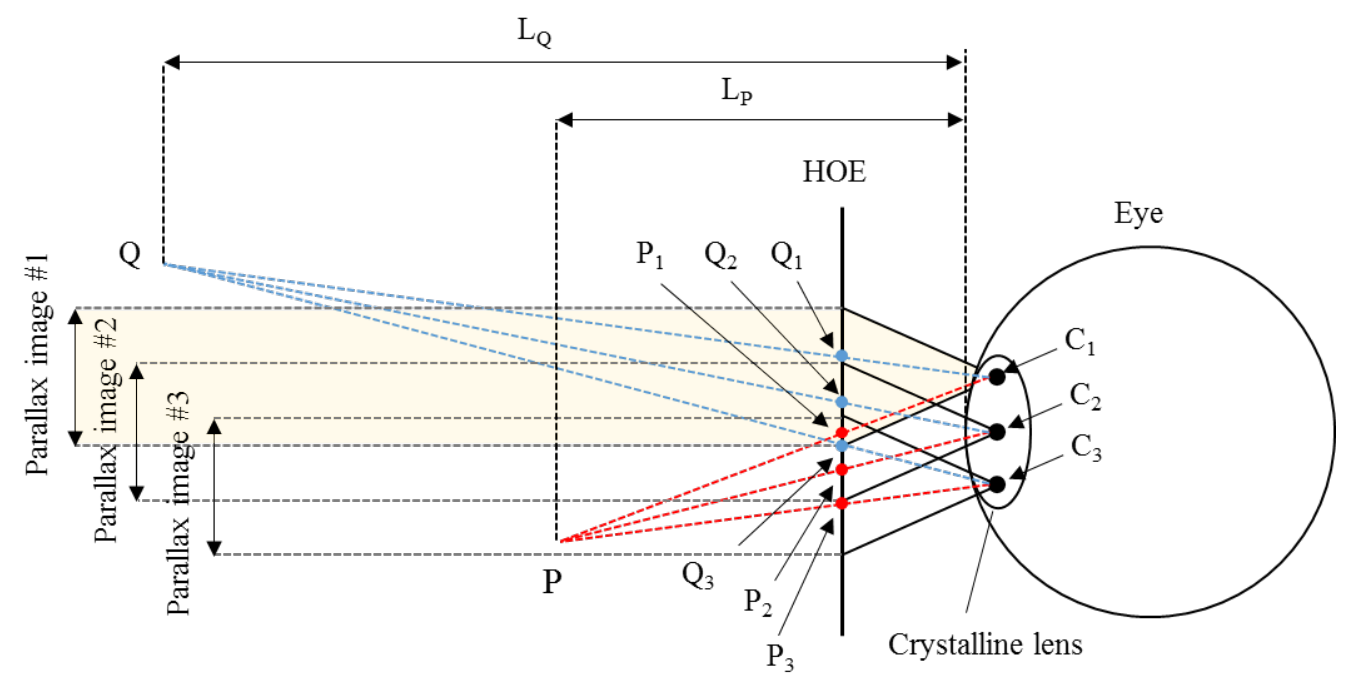

Figure 3. Schematic diagram of the proposed HMD. The super multi-view condition is realized by some projected parallax images which converge on the different positions on the pupil. 


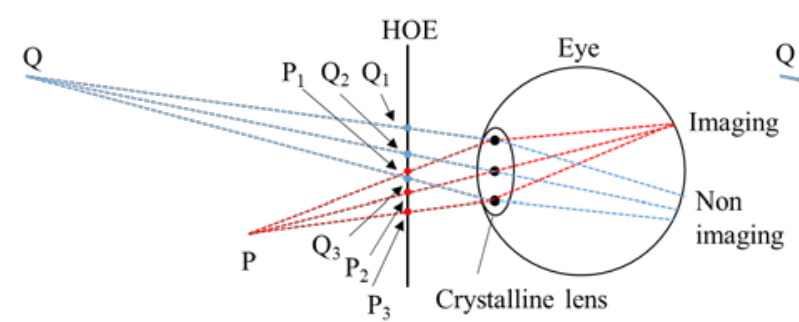

(a)

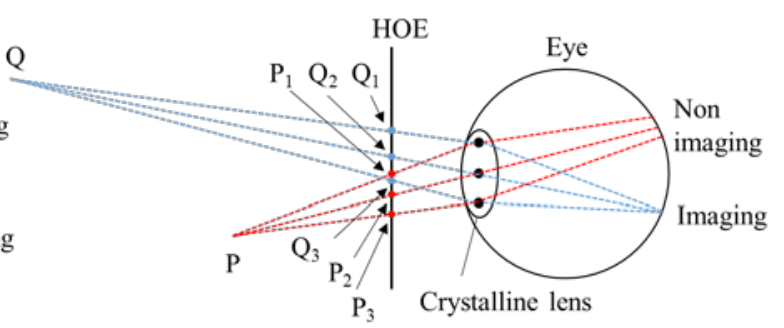

(b)

Figure 4. Principle of the monocular 3D HMD by using the super multi-view technique: (a) focusing on nearby point $\mathrm{P}$, (b) focusing on far-off point $\mathrm{Q}$.

\subsection{Retinal projection type super multi-view HMD by using the time division projection optical system}

We have previously proposed super multi-view HMD using time division projection optical system ${ }^{7}$. The proposed HMD consists of the multiple exposure holographic lens $\mathrm{HOE}_{2}$ which forms multiple convergence points, a high frame rate display device, a high speed shutter, and a holographic lens which works as a converging lens and a combiner. In this HMD, multiple parallax images are displayed by time division, and these images are converged on respective points by the multiple exposure holographic lens. The optical shutter which synchronized to the display device passes only one convergence light corresponding to the correct parallax image. Therefore, proposed HMD realizes the super multi-view condition. Figure 5 shows a schematic diagram of the retinal projection type super multi-view HMD using the time division projection optical system. In this figure, for the sake of simplicity, we limit the number of convergence points to three.

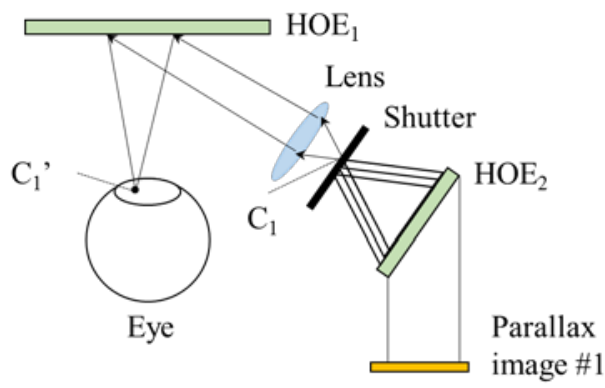

(a)

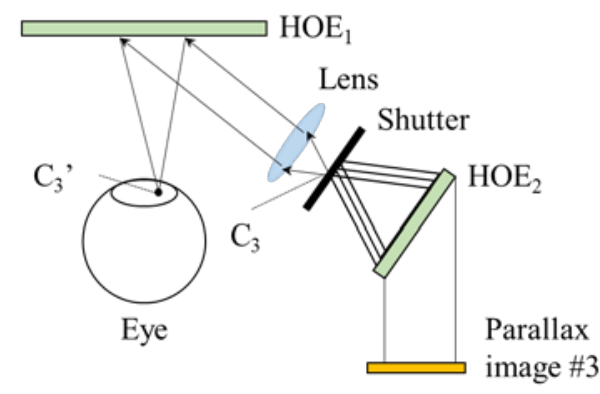

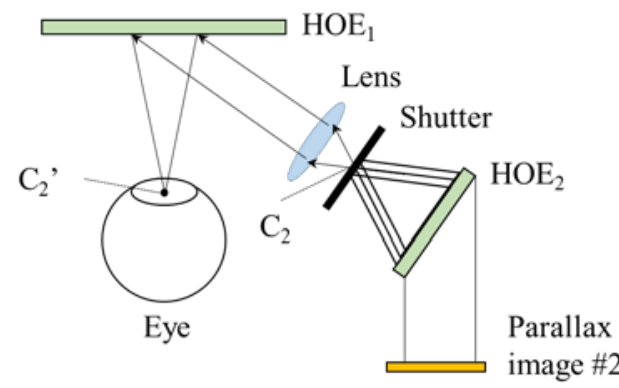

(b)

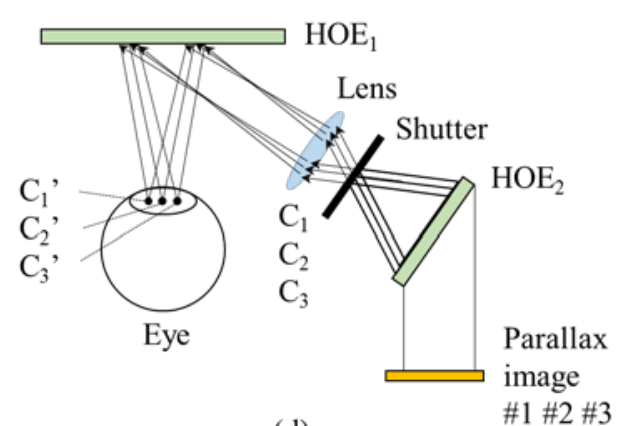

(c)

(d)

image

Figure 5. Schematic diagram of the proposed HMD based on the time division method with the high frame rate display device, the multiple exposure holographic lens and the high-speed optical shutter: (a) the optical system when only the convergence point $\mathrm{C}_{1}$ passes through the shutter, (b) the optical system when only the convergence point $\mathrm{C}_{2}$ passes through the shutter, (c) the optical system when only the convergence point $\mathrm{C}_{3}$ passes through the shutter, (d) the time division optical system works like the optical system as shown in Figure (d) by fast switching three states of Figure (a), (b) and (c) in one field. 
Since the eyeglass lens (holographic lens $\mathrm{HOE}_{1}$ ) of the HMD works as a convex lens, the conjugate images $\mathrm{C}_{1}$ ', $\mathrm{C}_{2}{ }^{\prime}$ and $\mathrm{C}_{3}{ }^{\prime}$ of the convergence points $\mathrm{C}_{1}, \mathrm{C}_{2}$ and $\mathrm{C}_{3}$ are located at the pupil, respectively. Since the conjugate images $\mathrm{C}_{1}{ }^{\prime}, \mathrm{C}_{2}{ }^{\prime}$ and $\mathrm{C}_{3}$ ' correspond to the parallax image \#1, \#2 and \#3 respectively, these parallax images projected on the retina. In Figure 5(a), only the convergence point $C_{1}$ passes through the shutter. In the same way, only the convergence point $C_{2}$ in Figure 5(b), and only the convergence point $\mathrm{C}_{3}$ in Figure 5(c), pass through the shutter respectively. In the proposed HMD, the time division optical system works like the optical system as shown in Figure 5(d) by fast switching three states of Figure 5(a), 5(b) and 5(c) in one field. So that these parallax images corresponding to each convergence point are left as afterimages on the human eye. Therefore, the super multi-view condition is realized with the time division optical system.

\section{RELATIONSHIP BETWEEN THE NUMBER OF PARALLAX IMAGES AND THE DISPLAYABLE DEPTH RANGE OF 3D IMAGE}

In the retinal projection type super multi-view 3D HMD, the super multi-view condition is realized by some parallax images which are projected on the retina. When a viewer focuses his eye on the displayed 3D image, he sees the in-focus image since multiple parallax images are superimposed at same position on the retina. On the other hand, when a viewer doesn't focus his eye on the 3D image, he sees the out-of-focus image since each parallax image is superimposed which is slightly displaced each other. Therefore, the stimulus for the accommodation of a human eye is produced naturally, and a viewer sees 3D images. However, if the amount of the position gap of multiple parallax images on the retina is large, the viewer cannot recognize superimposed parallax images as the blurred image. In this section, we will clarify the relationship between the number of parallax images and the displayable range of the 3D image by analyzing the maximum amount of position gap between corresponding pixels of the retinal image and the pixel size on the retina.

\subsection{The maximum amount of position gap between corresponding pixels of each parallax image on the retina}

We estimate the amount of position gap between corresponding pixels of each parallax image on the retina by using Gullstrand's schematic eye mode ${ }^{10}$, as shown in Figure 6. The definition of variables used in Figure. 6 are shown in Table 1. By using geometrical optics, we calculate the distance $\mathrm{W}$ between retinal image of each light ray and the optical axis. $v^{\prime}, D_{2}, v$, and $h_{2}$ can be obtained by

$$
\begin{gathered}
v^{\prime}=\frac{n}{D_{1}+\frac{1}{u}}, \\
D_{2}=\frac{n}{\left(L_{3}-L_{2}\right)}-\frac{n}{\frac{n}{\left(D_{1}+\frac{1}{d}\right)}-L_{2}}, \\
v=\frac{n}{\frac{n}{\left(v^{\prime}-L_{2}\right)}+D_{2}}
\end{gathered}
$$

and

$$
h_{2}=\frac{v^{\prime}-L_{2}}{v^{\prime}-L_{1}} h .
$$

From Eqs. (1), (2), (3), and (4), $W$ is given by

$$
W=\frac{h_{2}}{v}\left(v+L_{2}-L_{3}\right) .
$$

From Figure 7, the amount of position gap between corresponding pixels of each parallax image on the retina is equal to $W$. When a viewer focuses on the distance $d$ and a point light source is located at the distance $u(d>u), W$ becomes the maximum value within the range of the depth distance between $d$ and $u^{5}$. 


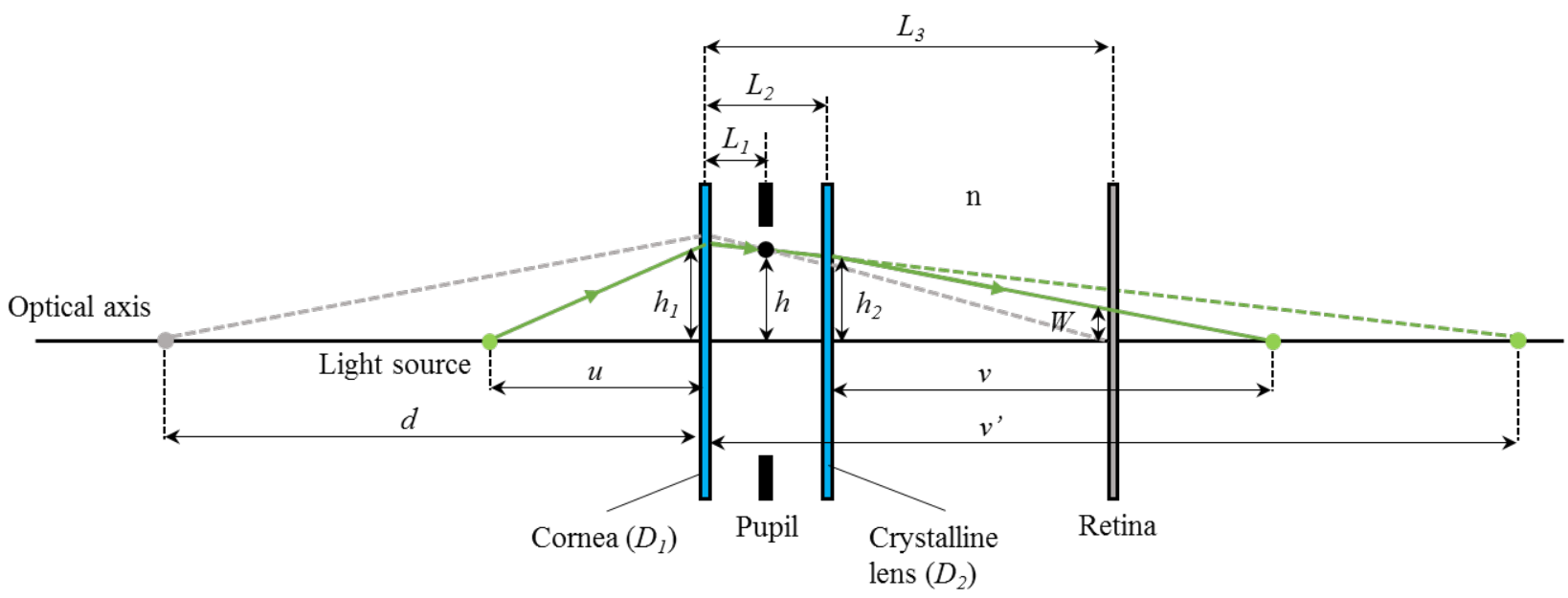

Figure 6. Gullstrand’s schematic eye model.

Table 1 . The definition of variables

\begin{tabular}{|c|c|}
\hline variable & meaning \\
\hline$u$ & Distance from cornea to object \\
\hline$v$ & Distance from the cornea to the imaging position \\
\hline$L_{1}$ & Distance from crystalline lens to imaging position \\
\hline$L_{2}$ & Distance from cornea to pupil \\
\hline$L_{3}$ & Distance from cornea to crystalline lens \\
\hline$h_{1}$ & Distance from cornea to retina \\
\hline$h_{2}$ & Height of ray on the cornea \\
\hline$W$ & Height of ray on the crystalline lens \\
\hline$h$ & Theight of ray on the retina \\
\hline$n$ & The refractive index in the eyeball pupil \\
\hline$d$ & Distance from the position where the observer focuses to \\
\hline$D_{1}$ & the cornea \\
\hline$D_{2}$ & Corneal refractive power \\
\hline \multicolumn{2}{|c|}{} \\
\hline
\end{tabular}

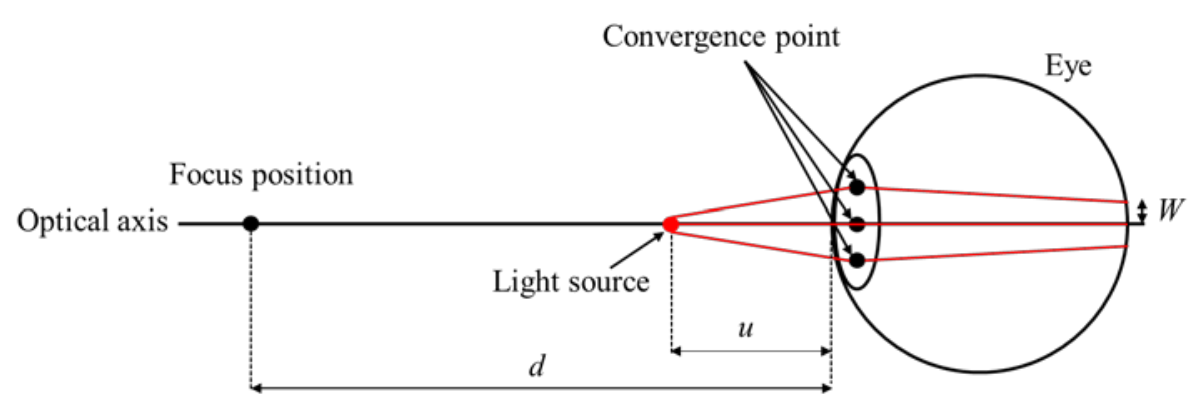

Figure 7. Schematic diagram of the maximum amount of position gap between corresponding pixels of the retinal image when a viewer focuses on the distance $d$. 


\subsection{Pixel size on the retina}

In order to calculate the pixel size on the retina, we analyze the light intensity distribution at the retina of the projected pixel by wave optics ${ }^{11}$. Figure 8 shows a schematic diagram of the retinal projection display and Figure 9 shows the geometry to calculate the light intensity distribution on the retina. In Figure $9, p$ is the pixel size, $z$ is the distance between the lens and the retina, $f$ is the focal length of the lens, and $\lambda$ is the wavelength. Referring to the geometry of Figure 9 , the lateral amplitude transfer function $\mathrm{h}(u)$ is derived by

$$
\begin{aligned}
& \mathrm{h}(u)=\mathrm{F} \cos \left\{\sqrt{\frac{\mathrm{k}}{\pi}\left|\frac{1}{\mathrm{z}}-\frac{1}{f}\right|}\left(\frac{p}{2}-\frac{x}{1-\frac{z}{f}}\right)\right\}+\mathrm{F} \cos \left\{\sqrt{\frac{\mathrm{k}}{\pi}\left|\frac{1}{z}-\frac{1}{f}\right|}\left(\frac{p}{2}+\frac{x}{1-\frac{z}{f}}\right)\right\} \\
& +\mathrm{j}\left[\mathrm{Fsin}\left\{\sqrt{\frac{\mathrm{k}}{\pi}\left|\frac{1}{\mathrm{z}}-\frac{1}{f}\right|}\left(\frac{p}{2}-\frac{x}{1-\frac{z}{f}}\right)\right\}+\mathrm{Fsin}\left\{\sqrt{\left.\left.\frac{\mathrm{k}}{\pi}\left|\frac{1}{z}-\frac{1}{f}\right|\left(\frac{p}{2}+\frac{x}{1-\frac{z}{f}}\right)\right\}\right],}\right.\right.
\end{aligned}
$$

where $\mathrm{k}$ is the wave number. $\mathrm{F} \cos (x)$ and $\mathrm{F} \sin (x)$ are the Fresnel cosine integral and Fresnel sine integral respectively and are defined as

$$
\mathrm{F} \cos (x)=\int_{0}^{x} \cos \left(\frac{\pi}{2} t^{2}\right) \mathrm{dt}
$$

and

$$
\operatorname{Fsin}(x)=\int_{0}^{x} \sin \left(\frac{\pi}{2} t^{2}\right) d t .
$$

Therefore, we can obtain the lateral light intensity distribution of the pixel in the retina $\mathrm{I}(u)$ by

$$
\mathrm{I}(u)=|\mathrm{h}(u)|^{2} \text {. }
$$

Let $p$ be $547 \mathrm{~nm}, z$ be $44 \mathrm{~nm}, f$ be $20 \mathrm{~mm}$, and $\lambda$ be $532 \mathrm{~nm}$, the light intensity distribution obtained as Figure 10 . In this figure, it is normalized by the light intensity at the center position on the retina. From Figure 10, the pixel size on the retina Ps can be obtained from the half width of the light intensity distribution.

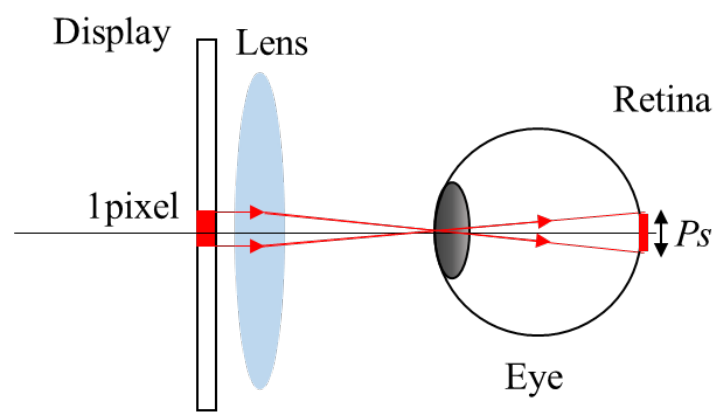

Figure 8. Schematic diagram of the retinal projection display. 


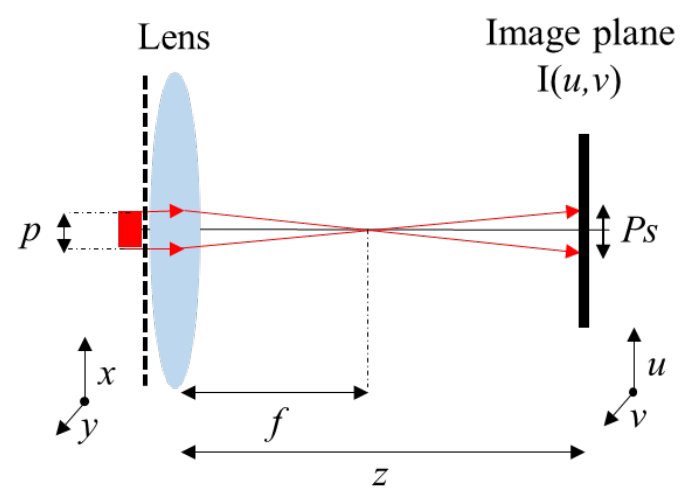

Figure 9. Geometry to calculate the light intensity distribution on the retina.

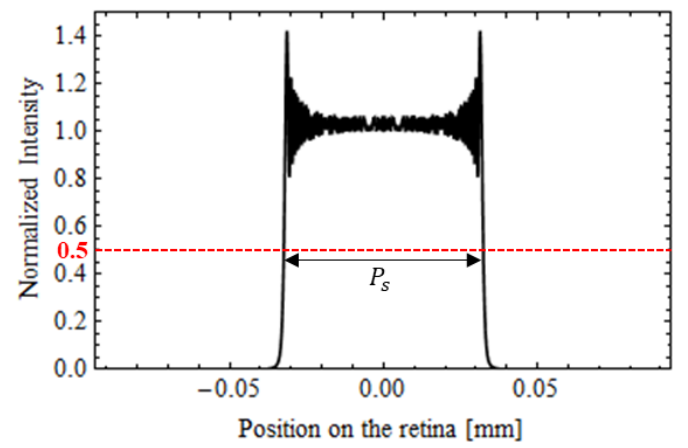

Figure 10. Schematic diagram of light intensity distribution on the retina.

\subsection{Relationship between the number of parallax images and the displayable depth range}

In the retinal projection type super multi-view 3D HMD, the super multi-view condition is realized by some parallax images which are projected on the retina. When a viewer focuses his eye on the displayed 3D image, he sees the in-focus image since multiple parallax images are superimposed at same position on the retina. On the other hand, when a viewer doesn't focus his eye on the 3D image, he sees the out-of-focus image since each parallax image is superimposed which is slightly displaced each other. Therefore, the stimulus for the accommodation of a human eye is produced naturally, and a viewer sees 3D images. However, if the amount of the position gap of multiple parallax images on the retina is large, the viewer cannot recognize superimposed parallax images as the blurred image. In this section, we will clarify the relationship between the number of parallax images and the displayable range of the 3D image by analyzing the maximum amount of position gap between corresponding pixels of each parallax image on the retina and the pixel size on the retina. For the sake of the simplicity, we consider the overlapping of two parallax images (parallax image \#1 and \#2). When the distance between left virtual 3D point and his eye is different from the distance between right virtual 3D point and his eye, two parallax images of left virtual 3D point are superimposed completely but them of right virtual 3D point are projected at different position on the retina, as shown in Figure 11. Figure 12 shows the light intensity distribution of the superimposed two parallax images projected on the retina when the observer views a virtual 3D point. When $W$ is less than or equal to $P s$, the retinal image is recognized as a blurred point. When $W$ is greater than $P$ s, since the retinal image is recognized as two points, the stimulus of the accommodation is not induced. Therefore, it is necessary to display a 3D image in the depth range where the retinal projection image has the condition $W>=P$ s. By using the maximum amount of position gap $W$ and the pixel size on the retina $P s$, it is possible to obtain a range that become a blurred point by

$$
P_{s} \geq \frac{2 W}{s-1}
$$


where s is the number of projected parallax images. In Eq. (10), since w and Ps are functions related to the display position of the virtual image, we can obtain the relationship between the number of parallax images and the displayable depth range.

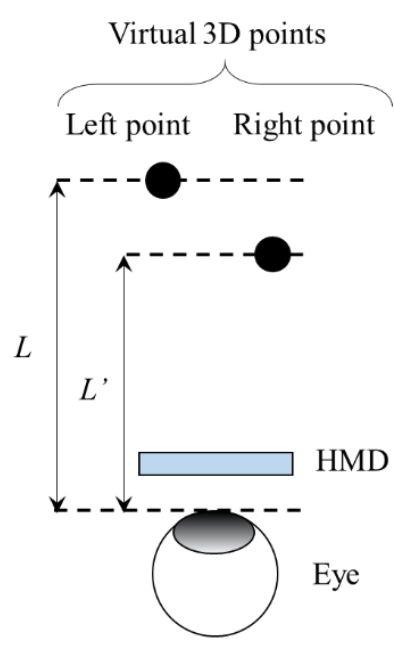

(a)

\section{Parallax image \#1}

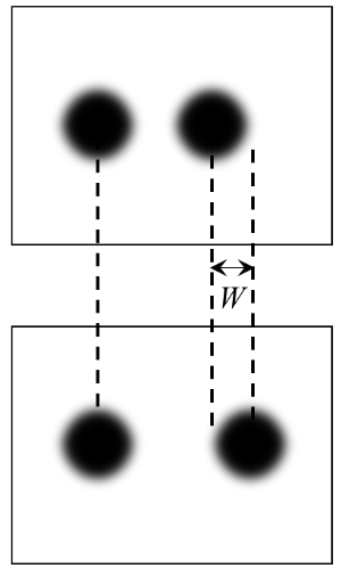

Parallax image \#2

(b)
Retinal image

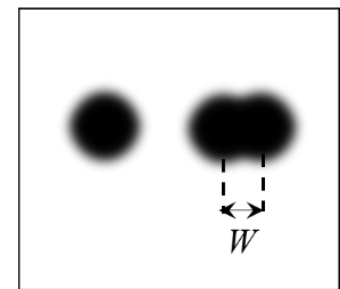

(c)

Figure 11. Retinal images when two points is displayed by the proposed HMD and a viewer focuses his eye on left point. The distance between two virtual 3D points and his eye is different: (a) schematic diagram of the location of virtual 3D points, (b) parallax images projected onto the retina, and (c) retinal image.

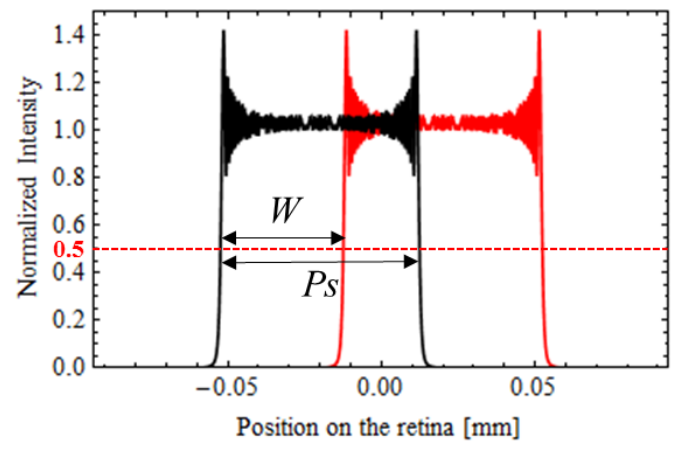

(a)

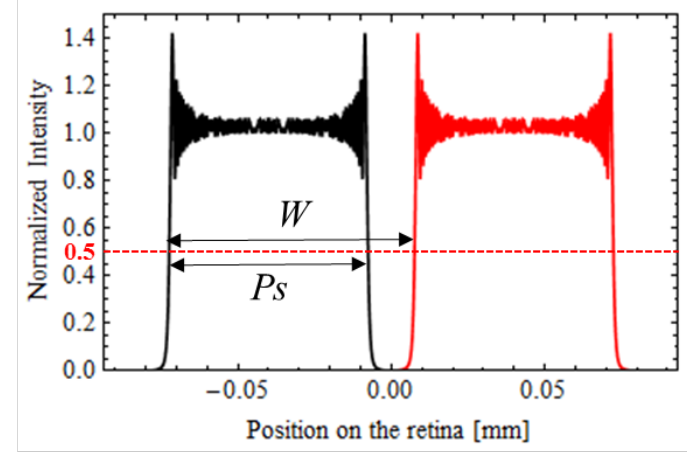

(b)

Figure 12. Schematic diagram of light intensity distribution on the retina when two point images are projected onto the retina: (a) W is smaller than Ps, (b) W is larger than Ps.

\section{EXPERIMENTAL RESULTS}

In order to verify the effectiveness of the proposed analysis method, we constructed the prototype system as shown in Figure 13. In the prototype system, the number of parallax images is five. The prototype system consists of a DMD, an eyeglass lens $\mathrm{HOE}_{1}$, a high-speed shutter, and a multiple exposure holographic lens $\mathrm{HOE}_{2}$. We used a DMD as a high frame rate display device which provides time sequential parallax images. In experiments, we used the camera as substitution for an eye and captured observed images. Since the camera was used instead of the eye, we set the eye relief of the eyeglass lens $\mathrm{HOE}_{1}$ to $100 \mathrm{~mm}$. In the prototype system, $p$ was $547 \mathrm{~mm}, z$ was $124 \mathrm{~mm}$, $f$ was $100 \mathrm{~mm}$, and $\lambda$ was $532 \mathrm{~nm}$. Figure 14 shows the schematic diagram of the experimental setup. We displayed the virtual images which is the vertically-striped pattern at the distance $900 \mathrm{~mm}$ in front of the camera, and captured observed image. Figures 15(a) and 
15(b) show the image which was taken with the camera in focus at a distance of $900 \mathrm{~mm}$ and $2000 \mathrm{~mm}$ in front of the camera, respectively. Figures 16(a) and 16(b) show the light intensity distribution of the observed images of Figures 15(a) and 15(b), respectively. As shown in Figures 15(a) and 16(a), the virtual vertically-striped image which was taken with the camera in focus at a distance of $900 \mathrm{~mm}$ in front of the camera could be observed clear. From Figures 15(b) and 16(b), the virtual image which was taken with the camera in focus at a distance of $2000 \mathrm{~mm}$ in front of the camera was not captured as multiple images and it was recognized as the blurred image. In Figure 16(a), the contrast of the light intensity distribution is $90.3 \%$. However, in Figure 15(b), the contrast is 69.1\%. In other words, when the camera is out of focus from the display position of the virtual 3D image, the contrast is lowered. Therefore, we confirmed that the retinal image in the prototype system can reconstruct the blur without becoming a multiple image. Therefore, it is shown that the relational expression between the number of parallax images and the displayable range of the 3D image is effective.

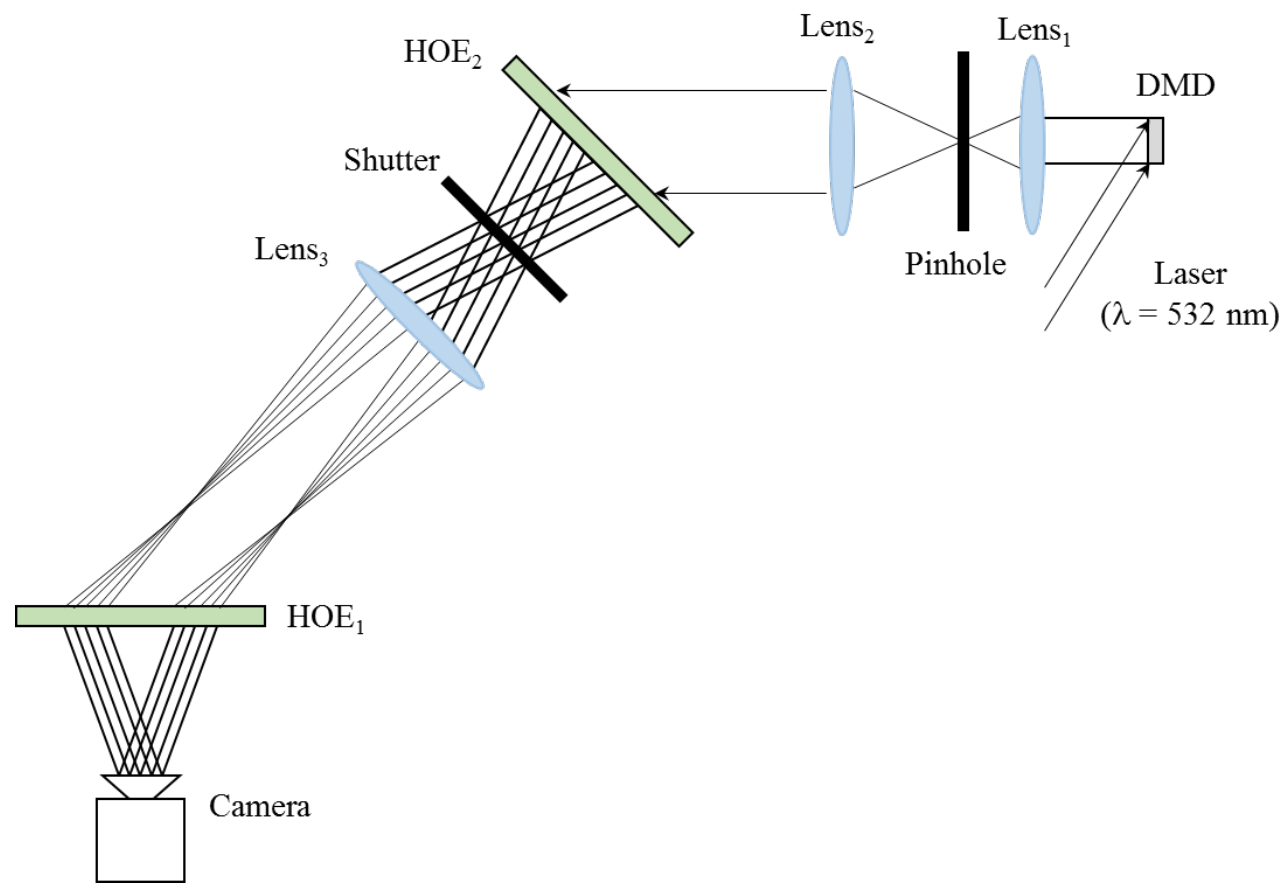

Figure 13. Prototype system.

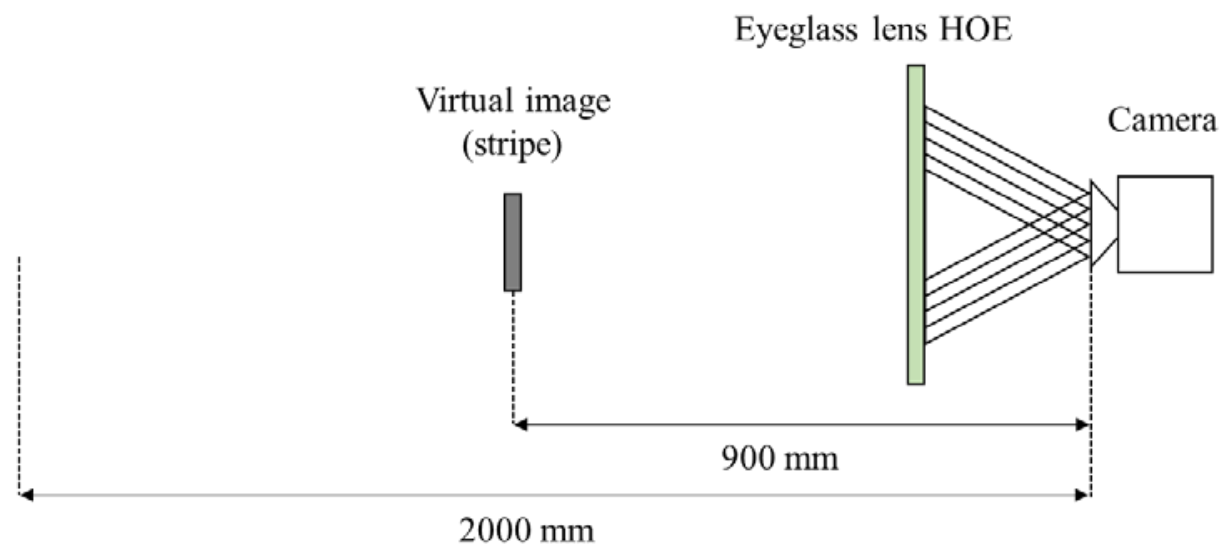

Figure 14. Schematic diagram of the experimental set up. 


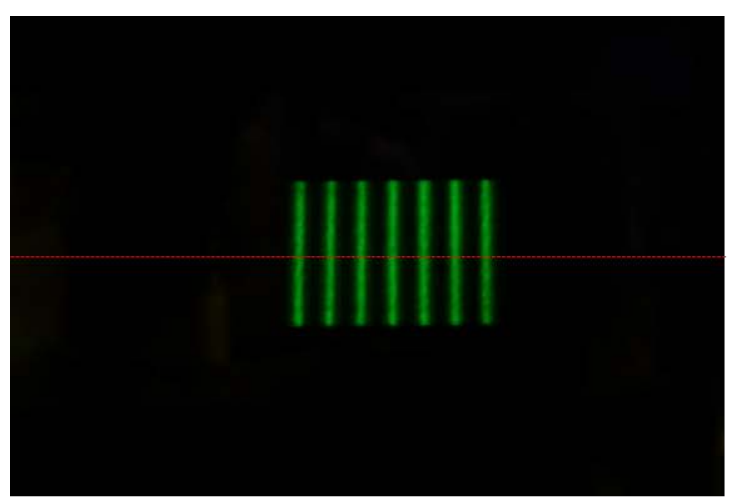

(a)

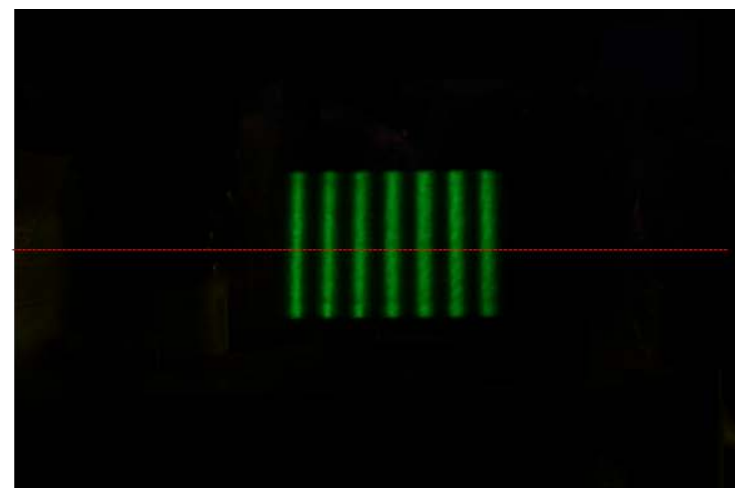

(b)

Figure 15. Observed images: (a) focused at the distance of $900 \mathrm{~mm}$, (b) focused at the distance of $2000 \mathrm{~mm}$.

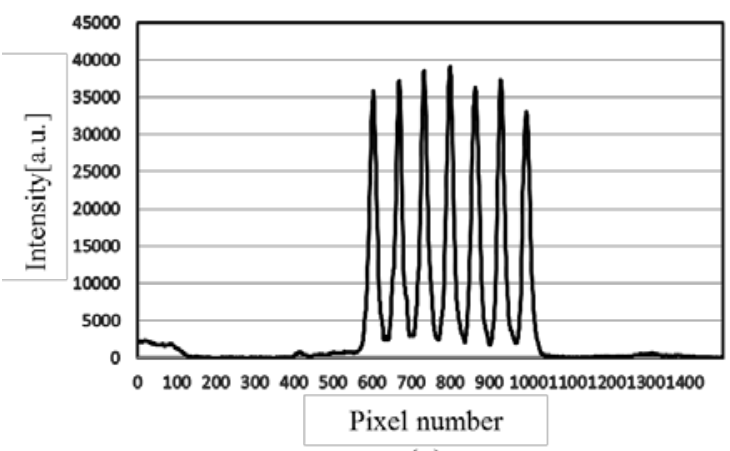

(a)

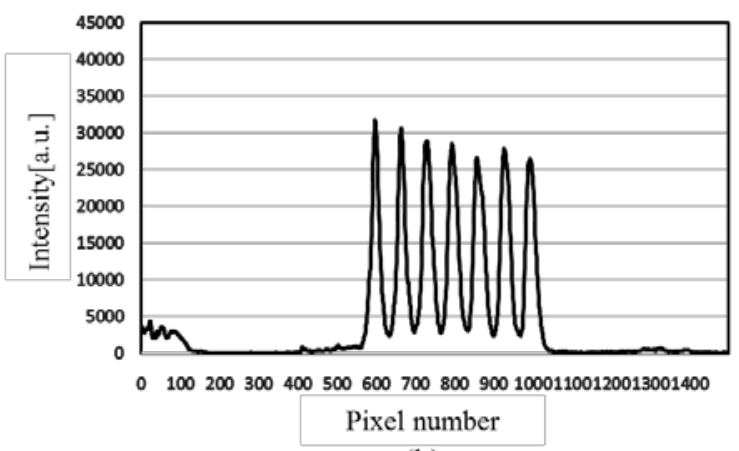

(b)

Figure 16. The light intensity distribution of the observed images: (a) focused at the distance of $900 \mathrm{~mm}$, (b) focused at the distance of $2000 \mathrm{~mm}$.

\section{CONCLUSION}

In this paper, we analyzed the relationship between the number of parallax images and the displayable depth range of the retinal projection type super multi-view 3D HMD. In order to verify the displayable range of the 3D image, we constructed the prototype HMD which projected five parallax images on the retina. The effectiveness of the proposed analysis method was confirmed by the experimental results of the displayable range of the prototype system. Therefore, by using the proposed analysis method, it is possible to freely design the displayable range of the 3D image of the super multi-view 3D HMD.

\section{ACKNOWLEDGEMENT}

This work was partially supported by JSPS KAKENHI Grant Number $18 \mathrm{H} 03277$. 


\section{REFERENCES}

[1] Tamura, H. and Ohta, V., [Mixed Reality], Ohmsha (1999).

[2] Burdea, G. and Coiffet, P., [Virtual Reality Technology], John Wiley \& Sons (1994).

[3] Takahashi, H., Ito, Y., Nakata, S. and Yamada, Y., "Retinal projection type super multi-view head-mounted display," Proc. SPIE-IS\&T Electronic Imaging, 9012, 90120L1-9012L6 (2014).

[4] Ito, Y., Takaichi, K. and Takahashi, H., "See-Through Retinal Projection Head-Mounted Display by using the Extended Maxwellian View," ICIC Express Letters 7(6), 1755-1760 (2013).

[5] Takatsuka, Y., Yoshimoto, K. and Takahashi, H., "Retinal projection type 3D head-mounted display using an HOE lens array," Proc. SPIE-IS\&T Electronic Imaging 2016, SDA-428.1-SDA-428.6 (2016).

[6] Emoto, T., Konda, T., Yoshimoto, K. and Takahashi, H., "Analysis of retinal images for retinal projection type super multi-view 3D head-mounted display," Proc. IS\&T Electronic Imaging 2017, 188-193 (2017).

[7] Konda, T., Tanaka, K., Yoshimoto, K. and Takahashi, H., "Retinal projection type super multi-view 3D headmounted display using the time division projection optical system," Proc. SPIE 10556, 105560L1-105560L11 (2018).

[8] Kajiki, Y., Yoshikawa, H. and Honda, T., "Ocular Accommodation by Super multi-view Stereogram and 45View Stereoscopic Display," Proc. IDW '96, 2, 489-492 (1996).

[9] Westheimer, G., "The Maxwellian View," Vision Res. 6, 669-682 (1966).

[10] Hermann, V. H., James, P. C. S., "Helmholtz’s Treatise on Phisiological Optics,” Thommes Press,Bristol(2000).

[11] Jae, H. P., Sung, W. M., Sungyong, J. and Byoungho, L., "Analysis of viewing parameters for two display methods based on integral photography," APPLIED OPTICS/Vol. 40, NO.29,pp.5217-5232 (2001). 